\title{
Genetic Analysis of New Maize Hybrids for Yield and Resistance to Aflatoxin Accumulation
}

\author{
Allen Oppong ${ }^{1}$, Abu M. Dadzie ${ }^{2}$, Beatrice Ifie ${ }^{3}$, Maxwell D. Asante ${ }^{1}$, Ruth N. A. Prempeh ${ }^{1}$, Linda A. Abrokwah ${ }^{1}$, \\ Zipporah Appiah Kubi ${ }^{1}$, Esther A. Marfo ${ }^{1}$, Esther A. Ananng ${ }^{1} \&$ Marilyn L. Warburton ${ }^{4}$ \\ ${ }^{1}$ CSIR-Crops Research Institute, Kumasi, Ghana \\ ${ }^{2}$ Cocoa Research Institute of Ghana, New Tafo-Akim Eastern Region, Ghana \\ ${ }^{3}$ West African Centre for Crop Improvement, College of Basic and Applied Sciences, University of Ghana, \\ Accra, Ghana \\ ${ }^{4}$ Corn Host Plant Resistance Research Unit (CHPRRU), United States Department of Agriculture, Agriculture \\ Research Service, MS, USA
}

Correspondence: Allen Oppong, CSIR-Crops Research Institute, Kumasi, Ghana. E-mail: alnopp@hahoo.co.uk

Received: June 28, 2021

doi:10.5539/jas.v13n10p15
Accepted: August 3, $2021 \quad$ Online Published: September 15, 2021

URL: https://doi.org/10.5539/jas.v13n10p15

\begin{abstract}
Maize (Zea mays L.) is the most important cereal crop in sub-Saharan Africa providing food for many of its inhabitants. However, production is hampered by low yields and aflatoxin contamination, among others. The toxin contaminates maize before harvest and during storage. Ghana lacks regulatory infrastructure for monitoring and detecting aflatoxin in grains prior to market, and most of the local maize varieties have been found to be susceptible to aflatoxin accumulation. Host resistance is envisaged as a key approach in addressing the aflatoxin menace. Thus, this study was conducted to identify new hybrids that combine high yield with resistance to aflatoxin accumulation as well as study the mode of gene controlling tolerance to aflatoxin accumulation. Sixteen aflatoxin resistant inbreds from exotic sources were crossed as males to 10 local germplasm in a North Carolina II design to generate 160 new hybrids. These were planted together with 9 checks using a $13 \times 13$ alpha lattice with three replications. The new hybrids were evaluated across six environments in two seasons. Five plants each per hybrid were inoculated with a local strain of Aspergillus flavus at a concentration of $9 \times 10^{7}$ conidia/ml. Ninety-six out of the 169 crosses were analyzed statistically. Significant effect of environment and genotypes for all traits especially, for aflatoxin accumulation resistance and yield were observed. The general combining ability effect of males for all traits were found significant $(\mathrm{P}<0.05)$ whereas that of the females were not significant for all traits. Inbreds with consistent significant negative GCA effect for aflatoxin reduction were identified (MP715, TZI8, MP719). Furthermore, the underlying genetic control for the aflatoxin accumulation resistance trait was found to be via both GCA and SCA effects. Heritability estimates were moderate, suggesting permissible transfer of traits during selection to create high yielding aflatoxin resistant hybrids for consumers.
\end{abstract}

Keywords: maize hybrids, aflatoxin contamination, maize yield, GCA/SCA, resistance to A. flavus

\section{Introduction}

The demand for maize continues to increase on the continent of Africa, as it accounts for an average of $32 \%$ of calories consumed in Eastern and Southern Africa, and up to 51\% in some countries (Cairns et al., 2012). Consumption of aflatoxin contaminated crops has been of health concern as this has been connected to malnutrition, immunosuppression, stunting or impaired child growth, kwashiorkor, liver cancer, acute aflatoxicosis, and death (Miller, 1996; Ramjee, 1996; Liang et al., 2006; Bankole et al., 2006; Gong et al., 2008; Matacic, 2016; Githang'a et al., 2019). Maize breeding programmes in Ghana have evolved from an initial use of local landrace varieties (which are poor in yield and nutrition) to the development of improved Open Pollinated Varieties (OPVs), many of which were bred as Quality Protein Maize (QPM), to the current era of hybrid variety production and promotion. Between $49 \%$ to $63 \%$ of the genetic gain in maize yields in Ghana has been attributed to the replacement of local varieties with improved varieties (Ewool, 2004; Kpotor, 2012). 
Current average yield of maize in Ghana is around 1.94-2.73 metric tons/ha (FAOSTAT, 2019; https://knoema.com/atlas/Ghana/topics/Agriculture/Crops-Production-Yield/Maize-yield). Further improvement to raise average yields to at least 4 metric tons/ha is needed to meet projected demand. Along with increases in yield, levels of aflatoxin accumulation have also been increasing, necessitating a program for improvement to resistance against aflatoxins. Previous varietal development in Ghana did not focus on aflatoxin resistance, as the serious nature of the problem was not understood. However, current surveys by Perrone et al. (2014) on harvested and consumed maize in Ghana revealed the presence of high levels of aflatoxin, well above approved recommended limits of the USA (20 ppb) and that of European Union (4 ppb). Availability of aflatoxin accumulation resistant hybrids will reduce aflatoxin contamination in maize and invariably help address in part the reported cases of serological contamination of over $98 \%$ of West African populations who consume contaminated maize and its products (Wild et al., 1990; Jolly et al., 2006; Agbetiameh et al., 2020).

To capitalize on heterosis in hybrid maize breeding requires the development of inbred parents with superior specific combining ability (SCA) for yield and disease resistance. Aflatoxin resistant inbred breeding lines have been developed by CIMMYT, IITA and the USDA, ARS, in Mississippi, USA; many of these lines originate from the Mexican landrace Tuxpêno (Warburton et al., 2014). These lines contain useful genetic variation for aflatoxin accumulation and A. flavus resistance breeding and can be used in testcrosses to identify productive and resistant hybrids.

Genetic variation associated with aflatoxin accumulation resistance is highly quantitative in nature, with many Quantitative Trait Loci (QTL) that has major effects on resistance. These QTL may act additively and may display high general combining ability (GCA) (Widstrom et al., 1984; Betran et al., 2002; Williams et al., 2008; Womack et al., 2020), which facilitate resistance breeding. Maize inbred lines with resistance to aflatoxin accumulation were identified in multi-environment field trials by Warburton et al. (2013). How these inbreds perform in Ghana, and when crossed to locally adapted inbred lines; and if these inbreds can produce high yielding hybrids for local cultivation, is unknown. Utilization of these resistant lines in Ghana for hybrid development could be direct, as parents of new hybrids, or may require transferring resistance genes into elite locally adapted genotypes. Thus, this study was conducted to identify new hybrid crosses that combine high yield with resistance to aflatoxin accumulation; to determine the combining abilities (GCA and SCA) and mode of gene action (additive, dominant, over dominant or epistatic) for genetic variation controlling tolerance to aflatoxin accumulation; and assess the performance of the hybrids across different growing environments in Ghana.

\section{Materials and Methods}

\subsection{Plant Materials}

The male germplasm used consisted of 16 inbred lines found to be resistant to aflatoxin accumulation over several locations and years by Warburton et al. (2013). They were obtained from the USDA ARS Corn Host Plant Resistance Research Unit, CIMMYT, and IITA and 10 additional genotypes that are well adapted to the Ghanaian environment and preferred by farmers (Table 1). The North Carolina design II was used to generate 160 hybrids at the CSIR-Crops Research Institute at Fumesua in 2016. The hybrids were evaluated with nine local checks; namely, CRI-ETUBI (hybrid) and CRI-MAMABA (hybrid), CRI-OMANKWA (OPV), CRI-TINTIM (hybrid), CRI-ABROHEMA (OPV), CRI-OBAATANPA (OPV), CRI-ABONTEM (OPV), CRI-AHOODZEN (OPV) and CRI-AFRIYIE (hybrid). 
Table 1. List of aflatoxin resistant inbred lines used as males and local germplasm used as females and origin/source

\begin{tabular}{ll}
\hline & Origin/Source \\
\hline Inbred Males & \\
CML11 & CIMMYT \\
CML158 & CIMMYT \\
CML176 & CIMMYT \\
CML247 & CIMMYT \\
CML287 & CIMMYT \\
CML322 & CIMMYT \\
CML343 & CIMMYT \\
CML5 & CIMMYT \\
Hi27 & THAILAND \\
Ki3 & THAILAND \\
MP705 & MISSISSIPI \\
MP715 & MISSISSIPI \\
MP719 & MISSISSIPI \\
NC298 & NORTH CAROLINA \\
NC356 & NORTH CAROLINA \\
Tzi8 & IITA \\
\hline Females & CIMMYT \\
ENTRY-5 & CIMMYT \\
ENTRY-6 & CIMMYT \\
ENTRY-70 & CIMMYT \\
ENTRY-85 & CIMMYT \\
GH-110 & IITA \\
\hdashline OMANKWA & IITA \\
M0826-12F* & \\
M0826-7F* & \\
TZEEI-15* & \\
\hline
\end{tabular}

\subsection{Experimental Sites and Field Layout}

The experiments were carried out in three locations all with a bimodal rainfall pattern that normally runs from March to July for the major season, and from September to November for the minor season. Fumesua is in the semi-deciduous forest zone at $286 \mathrm{~m}$ above sea level between $6.712 \mathrm{~N}$ and $1.523 \mathrm{~W}$. Rainfall averages $1500 \mathrm{~mm}$ in total, and temperatures range from $21-31{ }^{\circ} \mathrm{C}$. Soils around Fumesua are classified as Asuansi series, a ferric acrisol. Akomadan is within the forest savanna transition zone at $7.396 \mathrm{~N}, 1.973 \mathrm{~W}$ with temperature range of $20-35{ }^{\circ} \mathrm{C}$, with a rainfall ranging from $700 \mathrm{~mm}$ and $1200 \mathrm{~mm}$. Wenchi is located at $7.733 \mathrm{~N}, 2.100 \mathrm{~W}$ in the transitional savanna zone with temperature range of $21.2-31{ }^{\circ} \mathrm{C}$, with an annual rainfall of between about 1,140-1,270 $\mathrm{mm}$. The soil types are the savannah ochrosol with some lithosols (MoFA, 2020).

The experiments were planted in a $13 \times 13$ alpha lattice design during the major and minor seasons of 2017 in all three locations in three replications. Single row plots of $5 \mathrm{~m}$ spaced at $0.70 \mathrm{~m}$ apart with $0.4 \mathrm{~m}$ within plants in each row. Standard field agronomic practices were followed as needed.

\subsection{A. flavus Inoculation and Aflatoxin Measurements}

Pure cultures of A. flavus were obtained from local sources. L-type A. flavus as described by (Cotty, 1989; Klich \& Pitt, 1988) were maintained on potato dextrose agar (PDA) as described by Jha (1995). This toxigenic isolate was used to prepare inoculum as described by Windham et al. (2003). Briefly, the isolate was multiplied on sterile corn cob grit in 500-ml flasks each containing $50 \mathrm{~g}$ of grits and $100 \mathrm{ml}$ of sterile distilled water. This was incubated at $28{ }^{\circ} \mathrm{C}$ for 3 weeks. Conidia in each flask was then washed from the grits using $500 \mathrm{ml}$ of sterile distilled water containing 20 drops of Tween 20 per liter and then filtered through four layers of sterile 
cheesecloth. The concentrations of conidia determined with a hemocytometer and adjusted with sterile distilled water to $9 \times 10^{7}$ conidia per $\mathrm{ml}$.

The side needle technique described by Scott and Zummo (1994) was used to inoculate 5 plants/plot 14 days after mid-silk by inserting the needle under the husks on the upper $1 / 3$ of the ear and $3.4 \mathrm{ml}$ of a spore suspension of $9 \times 10^{7}$ conidia/ml was injected over the kernels. Approximately 60 days after mid-silk, cobs were harvested, dried shelled, and $50 \mathrm{~g}$ of samples ground using appropriate reagents for analysis using high-performance liquid chromatography (HPLC) to determine aflatoxin concentration levels as described by Wacco et al. (2014).

\subsection{Data Collection}

In addition to aflatoxin levels, data were collected on 15 traits: days to $50 \%$ silking; days to $50 \%$ pollen shed; anthesis-silking interval; ear aspect; plant aspect; root lodging; stalk lodging; ear number per plant; husk cover; stay-green; maize blight resistance; maize streak resistance; grain moisture content; field weight and grain yield ( $\mathrm{kg} / \mathrm{ha}$ ), which was determined at 15\% moisture content according to Barreto and Raun (1988) as:

$$
\text { Grain Yield }=\frac{\text { Field Weight }(\mathrm{kg})}{\text { Harvested Area }\left(\mathrm{m}^{2}\right)} \times \frac{10000\left(\mathrm{~m}^{2}\right)}{\mathrm{ha}} \times \frac{100 \% \text { - Grain Moisture }(\%)}{85} \times 0.8 \times \text { Shelling }(\%)
$$

\subsection{Statistical Analysis}

Analysis of Variance (ANOVA) was performed on plot means for grain yield and all other agronomic traits across environments using the PROC GLM procedure of the SAS software package, version 9.4 (SAS Institute, 2012). Data on aflatoxin contamination was natural $\log$ transformed and data for ear rot, stalk and root lodging percentages were transformed using arcsine. Genotype or entry means were adjusted for block effects and analysed according to alpha lattice design (Cochran \& Cox, 1960). Each environment was defined as season $\times$ site $\times$ aflatoxin inoculation treatment. Effects of environment were considered as random while genotypes were classified as fixed.

The hybrids component of variation was divided into variation due to male, female, and female $\times$ male interaction. Male and female main effects represent general combining ability (GCA) while female $\times$ male interaction represents specific combining ability (SCA) effect (Hallauer \& Miranda, 1988). The F tests for male, female, and female $\times$ male mean squares were computed using the mean squares for their respective interaction with environment. The mean square attributable to environment $\times$ female $\times$ male was tested using the pooled error mean squares. The general linear model for the NC II mating design used was:

$$
\mathrm{Y}_{\mathrm{ijk}}=\mu+\mathrm{M}_{\mathrm{i}}+\mathrm{F}_{\mathrm{j}}+\mathrm{MF}_{\mathrm{ij}}+\mathrm{R}_{\mathrm{k}}+\varepsilon_{\mathrm{ijk}}
$$

Where, $Y_{\mathrm{ijk}}=$ observed trait value; $\mu=$ Mean effect; $M_{i}=$ Effect of the $i^{\text {th }}$ male; $F_{j}=$ Effect of the $j^{\text {th }}$ female; $M_{i j}$ $=$ Effect of interaction between $\mathrm{i}^{\text {th }}$ male and $\mathrm{j}^{\text {th }}$ female; $\mathrm{R}_{\mathrm{k}}=$ Effect of $\mathrm{K}^{\text {th }}$ replication; $\mathrm{d} \varepsilon_{\mathrm{ijk}}=$ experimental error.

Restricted maximum likelihood (REML) estimates of the inbreds' and hybrids' genetic and phenotypic variances were obtained with SAS PROC variance components and were used to compute broad-sense heritability $\left(\mathrm{H}^{2}\right)$ for each trait as;

$$
\mathrm{h}^{2}=\sigma_{\mathrm{G}}^{2} /\left(\sigma_{\mathrm{E}}^{2} / \mathrm{re}+\sigma_{\mathrm{GE}}^{2} / \mathrm{e}+\sigma_{\mathrm{G}}^{2}\right)
$$

Where, $\sigma_{G}^{2}$ is variance for differences in phenotype due to genotype; $\sigma_{\mathrm{E}}^{2}$ is error variance, $\sigma_{\mathrm{GE}}^{2}$ is genotype $\times$ environment interaction variance; $r$ is number of replications; e is number of environments (Fehr, 1991). Correlation coefficients were computed between grain yield and other agronomic traits using the PROC CORR from SAS.

Estimates of GCA and SCA were calculated and their significance determined by $t$ tests. Male and female general combining ability was estimated as:

$$
\mathrm{GCA}_{\mathrm{f}}=\mathrm{X}_{\mathrm{f}}-\mu \text {, and } \mathrm{GCA}_{\mathrm{m}}=\mathrm{X}_{\mathrm{m}}-\mu
$$

Where, $\mathrm{GCA}_{\mathrm{m}}$ and $\mathrm{GCA}_{\mathrm{f}}=$ General combining ability of male and female parents respectively; $\mathrm{X}_{\mathrm{f}}$ and $\mathrm{X}_{\mathrm{m}}=$ Mean of male and female parents respectively; $\mu=$ Overall mean of crosses in the trial.

Specific combining ability (SCA) was estimated as:

$$
\mathrm{SCA}_{\mathrm{X}}=\mathrm{X}_{\mathrm{X}}-\mathrm{E}\left(\mathrm{X}_{\mathrm{X}}\right)=\mathrm{X}_{\mathrm{X}}-\left[\mathrm{GCA}_{\mathrm{f}}+\mathrm{GCA}_{\mathrm{m}}+\mu\right]
$$

Where, $\mathrm{SCA}_{\mathrm{x}}=$ specific combining ability of the cross $\mathrm{x} ; \mathrm{X}_{\mathrm{X}}=$ Observed mean value of the cross; $\mathrm{E}\left(\mathrm{X}_{\mathrm{X}}\right)=$ Expected mean value of the cross based on the GCA of both parent; $\mu=$ Overall mean of crosses.

The relative importance of GCA effects versus SCA effects on progeny performance was calculated as the ratio 
between sum of square due to GCA to the total genotypic sum of squares (GCA and SCA sum of square) (Beck et al., 1990; Pswarayi \& Vivek, 2008). Estimates of GCA of each male were obtained based on its performance in F1 hybrid combinations with all possible females. GCA and SCA effects were determined for each agronomic trait across all environments.

The mid-parent heterosis (MPH) and better parent heterosis (BPH) values for a cross was computed for aflatoxin levels and yield/ha as:

$$
\mathrm{MPH}=\left[\left(\mathrm{F}_{1}-\mathrm{MP}\right) / \mathrm{MP}\right] \times 100 \text {, and } \mathrm{BPH}=\left[\left(\mathrm{F}_{1}-\mathrm{BP}\right) / \mathrm{BP}\right] \times 100
$$

Where, $\mathrm{F}_{1}=$ Mean of the hybrid; $\mathrm{MP}=$ the mean of the parents that constituted the hybrids; $\mathrm{BP}=$ the mean of the better parent; MPH and BPH were averaged across aflatoxin contaminated environment.

A modified base index based on the selection index proposed by Badu-Apraku et al. (2011) was formulated to rank the hybrids as follows:

$$
\text { Base index }=[2.0 \mathrm{GY}+\mathrm{EPP}-\mathrm{SG}-\mathrm{CA}-\mathrm{PA}-\ln (\mathrm{Y}+1)-\mathrm{ER}]
$$

Where, $\mathrm{GY}=$ Grain yield; $\mathrm{SG}=$ Stay green; $\mathrm{CA}=$ Cob aspects; $\mathrm{PA}=$ Plant Aspects; $\ln (\mathrm{Y}+1)=$ Aflatoxin levels; $\mathrm{ER}=$ Ear rot.

\section{Results}

\subsection{Combined Genetic Analysis of Traits Across Six Environments}

Statistical analysis of agronomic and aflatoxin resistance traits across the six environments for 96 hybrids are presented in Table 2. The analysis shows significant effects of environment and genotypes for all traits and the effect of replication within each environment. The general combining ability effect of lines used as males for all traits was significant $(\mathrm{P}<0.05)$ whereas those used as females was significant for some of the traits (Table 2).

\subsection{General Combining Ability Estimates of Inbred Lines for Traits}

The general combining ability estimates for grain yield was positive and significant only for inbred line NC298 used as a male (1176.82), which is indicative of a good combiner (Table 3). Two inbreds, MP715 and Tzi8, displayed significant negative GCA for aflatoxin accumulation resistance (where negative is the desired outcome) when used as males, but all exotic lines except CML5, CML343 and Hi27 displayed negative but non-significant GCA for aflatoxin reduction when used as males (Table 3). General combining ability estimates for the other traits for all inbred lines are indicated in Table 3.

Table 2. Mean squares of 96 maize hybrids evaluated across six environments during the major and minor

\begin{tabular}{|c|c|c|c|c|c|c|c|c|c|c|}
\hline Sources of variation & $\begin{array}{l}\mathrm{DF} \\
\text { (Agronomic } \\
\text { traits) }\end{array}$ & $\begin{array}{l}\text { Grain Yield } \\
(\mathrm{kg} / \mathrm{ha})\end{array}$ & $\begin{array}{l}\text { Days to } \\
(50 \% \text { pollen })\end{array}$ & $\begin{array}{l}\text { Days to } \\
\text { (50\% Silking) }\end{array}$ & $\begin{array}{l}\text { Anthesis } \\
\text { Silking } \\
\text { Interval }\end{array}$ & $\begin{array}{l}\text { Ears Per } \\
\text { Plant }\end{array}$ & $\begin{array}{l}\text { Cob } \\
\text { Aspect } \\
(1-5)\end{array}$ & $\begin{array}{l}\text { Plant } \\
\text { Aspect }\end{array}$ & $\begin{array}{l}\text { Df (Aflatoxin } \\
\text { accumulation) }\end{array}$ & $\begin{array}{l}\text { Aflatoxin } \\
\ln (Y+1)\end{array}$ \\
\hline ENV & 5 & $523129824.86^{* * *}$ & $1693.81 * * *$ & $1627.20 * * *$ & $16.83 * * *$ & $4.71 * * *$ & $44.99 * * *$ & $80.80^{* * *}$ & 5 & $119.7 * * *$ \\
\hline REP(ENV) & 12 & $2752243.26^{* * *}$ & $56.39^{* * *}$ & $61.44 * * *$ & $2.14^{* * *}$ & $0.07 * * *$ & $0.42^{* * *}$ & $1.77^{* * *}$ & 6 & $0.30^{* * *}$ \\
\hline GENOTYPES & 95 & $13081077.69^{* * *}$ & $83.01 * * *$ & $85.48 * * *$ & $2.32 * * *$ & $0.15^{*}$ & $0.88^{* * *}$ & $1.02 * * *$ & 63 & $3.10^{* * *}$ \\
\hline MALE (GCAm) & 15 & $31932002.02 * * *$ & $170.85^{* * *}$ & $190.67 * * *$ & $3.04 * * *$ & $0.24 *$ & $2.56^{* * *}$ & $2.78^{* * * *}$ & 15 & $2.50 * * *$ \\
\hline FEMALE (GCAf) & 5 & $32981333.71 * * *$ & $781.87 * * *$ & $756.67 * * *$ & $5.36^{* * *}$ & $0.27 *$ & $0.32 \mathrm{~ns}$ & $1.18^{*}$ & 3 & $0.90^{*}$ \\
\hline MALE:FEMALE (SCA) & 75 & $7978464.20^{* * *}$ & $18.87 \mathrm{~ns}$ & $19.74 \mathrm{~ns}$ & $1.98^{* * *}$ & $0.12 \mathrm{~ns}$ & $0.58 * * *$ & $0.66^{* *}$ & 45 & $3.20^{* * *}$ \\
\hline ENV:GENOTYPES & 475 & $5655396.45^{* * *}$ & $19.76^{*}$ & $21.11 \mathrm{~ns}$ & $1.16^{* * *}$ & $0.12 *$ & $0.41^{* *}$ & $0.68^{* * *}$ & 315 & $2.05^{* * *}$ \\
\hline ENV:MALE (GCAm) & 75 & $10220890.40^{* * *}$ & $22.28 *$ & $25.34 *$ & $1.75^{* * *}$ & $0.19 *$ & $0.58 * * *$ & $0.85 * * *$ & 75 & $2.2 * * *$ \\
\hline ENV:FEMALE (GCAf) & 25 & $15217316.09^{* * *}$ & $33.56^{*}$ & $39.94 * *$ & $2.13^{* * *}$ & $0.18^{*}$ & $0.83 * * *$ & $1.32 * * *$ & 15 & $1.34 * * *$ \\
\hline ENV:MALE:FEMALE (SCA) & 375 & $4106391.79^{* * *}$ & $18.36 \mathrm{~ns}$ & $19.04 \mathrm{~ns}$ & $0.98^{* *}$ & $0.11 \mathrm{~ns}$ & $0.35 \mathrm{~ns}$ & $0.60^{* * *}$ & 225 & $2.10^{* *}$ \\
\hline Pooled error & 907 & 2126127 & 16.74 & 18.63 & 0.74 & 0.11 & 0.32 & 0.43 & 228 & 0.24 \\
\hline Broad Sense $\left(\mathrm{H}^{2}\right)$ & & 53.93 & 48.70 & 46.30 & 41.90 & 10.50 & 35.20 & 27.50 & & 72.00 \\
\hline
\end{tabular}
seasons of 2017

Note. ${ }^{*}, * * *=$ significant at 0.01 and 0.05 respectively, $\mathrm{ns}=$ not significant. $\mathrm{H}^{2}=$ broad sense heritability; Env $=$ environment; Rep $=$ replication; GCA $=$ General combining ability; SCA $=$ Specific combining ability. 
Table 2. Continued

\begin{tabular}{|c|c|c|c|c|c|c|c|c|}
\hline Sources of variation & $\mathrm{DF}$ & $\begin{array}{l}\text { Husk } \\
\text { Cover } \\
(1-5)\end{array}$ & Stay-Green & $\begin{array}{l}\text { Insect } \\
\text { Damage } \\
(1-5)\end{array}$ & $\begin{array}{l}\text { Rust } \\
\text { Disease } \\
(1-5)\end{array}$ & $\begin{array}{l}\text { Blight } \\
\text { Disease } \\
(1-5)\end{array}$ & $\begin{array}{l}\text { Maize streak } \\
\text { virus disease } \\
(1-5)\end{array}$ & $\begin{array}{l}\text { Ear rot } \\
(1-5)\end{array}$ \\
\hline ENV & 5 & $41.28 * * *$ & $321127.17 * * *$ & $15.52 * * *$ & $2.76 * * *$ & $7.49 * * *$ & $106.93 * * *$ & $17.94 * * *$ \\
\hline REP (ENV) & 12 & $0.49 * * *$ & $25973.29 * * *$ & $0.32 * * *$ & $0.33 * * *$ & $0.43 * * *$ & $1.33 * * *$ & $2.03 * * *$ \\
\hline GENOTYPES & 95 & $0.32 * * *$ & $209889.80 * * *$ & $0.26 * * *$ & $0.43 * * *$ & $0.52 * * *$ & $2.35 * * *$ & $0.40 * * *$ \\
\hline MALE (GCAm) & 15 & $0.34 *$ & $192926.76^{* * *}$ & $0.43 * * *$ & $1.27 * * *$ & $0.98 * * *$ & $5.24 * * *$ & $0.75^{* * *}$ \\
\hline FEMALE (GCAf) & 5 & $0.361 \mathrm{~ns}$ & $384130.44 * * *$ & $0.40 * *$ & $1.16^{* * *}$ & $2.45^{* * *}$ & $17.63 * * *$ & $0.41 \mathrm{~ns}$ \\
\hline MALE:FEMALE (SCA) & 75 & $0.31 * *$ & $202578.82 * * *$ & $0.21 * * *$ & $0.20 *$ & $0.29 * * *$ & $0.75 * * *$ & $0.33^{*}$ \\
\hline ENV:GENOTYPES & 475 & $0.28 * * *$ & $99857.35^{* * *}$ & $0.19 * * *$ & $0.24 * * *$ & $0.28 * * *$ & $0.69 * * *$ & $0.27 *$ \\
\hline ENV:MALE (GCAm) & 75 & $0.28 * *$ & $94704.22 * *$ & $0.22 * * *$ & $0.39 * * *$ & $0.55 * * *$ & $0.85 * * *$ & $0.33^{*}$ \\
\hline ENV:FEMALE (GCAf) & 25 & $0.41 * *$ & $144657.66^{* * *}$ & $0.25 * *$ & $0.26^{*}$ & $0.51 * * *$ & $0.84 * *$ & $0.21 \mathrm{~ns}$ \\
\hline ENV:MALE:FEMALE (SCA) & 375 & $0.27 * *$ & $97700.02 * * *$ & $0.19 \mathrm{~ns}$ & $0.20 *$ & $0.21 * *$ & $0.65 * * *$ & $0.26 * * *$ \\
\hline Pooled error & 907 & 0.18 & 55287.68 & 0.12 & 0.16 & 0.16 & 0.41 & 0.23 \\
\hline Broad Sense $\left(\mathrm{H}^{2}\right)$ & & 22.00 & 49.10 & 27.00 & 29.20 & 37.70 & 52.10 & 21.10 \\
\hline
\end{tabular}

Note. ${ }^{*}, * *=$ significant at 0.01 and 0.05 respectively, $\mathrm{ns}=$ not significant. $\mathrm{H}^{2}=$ broad sense heritability.

Table 3. GCAs of lines involved in the development of hybrids

\begin{tabular}{|c|c|c|c|c|c|c|c|}
\hline Lines & Grain Yield & $\begin{array}{l}\text { Days to } \\
\text { (50\% Pollen) }\end{array}$ & $\begin{array}{l}\text { Days to } \\
\text { (50\% Silking) }\end{array}$ & $\begin{array}{l}\text { Anthesis Silking } \\
\text { Interval }\end{array}$ & Ear per Plant & Cob Aspect & Plant Aspect \\
\hline \multicolumn{8}{|l|}{$M A L E G C A$} \\
\hline CML11 & 702.84 & 0.84 & 0.97 & 0.20 & 0.01 & -0.11 & 0.03 \\
\hline CML158 & -197.92 & 1.01 & 0.91 & -0.08 & -0.03 & -0.17 & -0.03 \\
\hline CML176 & 7.07 & 0.56 & 0.46 & -0.09 & -0.03 & -0.14 & -0.03 \\
\hline CML247 & 341.68 & -0.55 & -0.59 & -0.01 & 0.07 & -0.16 & -0.06 \\
\hline CML287 & 549.25 & 2.15 & 2.06 & -0.09 & 0.00 & -0.03 & -0.09 \\
\hline CML322 & 448.75 & -0.12 & -0.25 & -0.11 & -0.01 & -0.09 & -0.16 \\
\hline CML343 & -90.73 & 0.77 & 0.95 & 0.19 & $0.16^{*}$ & -0.15 & -0.20 \\
\hline CML5 & 112.93 & 0.28 & 0.22 & -0.05 & $0.13^{*}$ & -0.02 & 0.02 \\
\hline Hi27 & -351.89 & -1.23 & -1.30 & -0.08 & -0.06 & 0.20 & $0.32 *$ \\
\hline Ki3 & 377.15 & -1.76 & -1.92 & -0.13 & 0.00 & 0.08 & 0.00 \\
\hline MP705 & -964.5 & -1.47 & -1.16 & $0.33^{*}$ & -0.05 & 0.23 & 0.28 \\
\hline MP715 & -37.41 & $2.62 *$ & $2.95^{*}$ & 0.09 & -0.02 & 0.24 & 0.06 \\
\hline MP719 & -4.18 & 0.78 & 0.99 & 0.20 & -0.08 & 0.16 & -0.01 \\
\hline NC298 & $1176.82 *$ & -1.07 & -1.36 & -0.27 & 0.01 & 0.20 & 0.14 \\
\hline NC356 & -528.19 & -1.44 & -1.31 & 0.12 & -0.03 & 0.11 & 0.15 \\
\hline TZI8 & 748.11 & -0.92 & -1.18 & -0.25 & 0.04 & -0.20 & $0.34 *$ \\
\hline $\mathrm{SE} \pm$ & 526.00 & 1.22 & 1.30 & 0.16 & 0.10 & 1.50 & 0.16 \\
\hline \multicolumn{8}{|c|}{ FEMALE GCA } \\
\hline ENTRY-5 & 219.43 & 1.22 & 0.96 & -0.24 & 0.01 & 0.01 & 0.07 \\
\hline Entry-6 & -710.13 & 1.21 & 1.21 & 0.00 & -0.05 & 0.01 & 0.06 \\
\hline ENTRY-70 & 118.3 & 1.39 & 1.55 & 0.15 & -0.02 & 0.01 & -0.01 \\
\hline ENTRY-85 & 263.41 & 0.57 & 0.60 & 0.06 & 0.02 & 0.04 & 0.04 \\
\hline TZEEI-15 & -16.59 & -2.13 & -2.12 & -0.06 & 0.00 & -0.05 & -0.09 \\
\hline TZEEI-6 & 101.63 & -2.11 & -2.04 & 0.09 & 0.04 & 0.05 & -0.05 \\
\hline $\mathrm{SE} \pm$ & 308.92 & 1.50 & 1.50 & 0.12 & 0.03 & 0.03 & 0.05 \\
\hline
\end{tabular}

Note. ${ }^{*}, * *$ Significant at 0.05 and 0.01 probability levels. $\mathrm{SE} \pm$ : standard error. 
Table 3. Continued

\begin{tabular}{|c|c|c|c|c|c|c|c|c|}
\hline Lines & Aflatoxin $\ln (\mathrm{Y}+1)$ & Husk Cover & Stay-Green & Insect Damage & Leaf Rust & Leaf Blight & Maize Streak & Ear rot \\
\hline \multicolumn{9}{|l|}{ MALE GCA } \\
\hline CML11 & -0.01 & $0.13 *$ & $149.62 * * *$ & 0.03 & 0.10 & 0.02 & 0.31 & -0.07 \\
\hline CML158 & -0.04 & -0.07 & -13.39 & -0.05 & -0.02 & 0.01 & -0.26 & -0.06 \\
\hline CML176 & -0.03 & 0.01 & -14.36 & -0.09 & 0.07 & 0.13 & 0.15 & 0.01 \\
\hline CML247 & -0.07 & 0.07 & -13.21 & $0.13^{* *}$ & -0.10 & -0.12 & -0.04 & -0.05 \\
\hline CML287 & -0.02 & 0.04 & -13.05 & 0.04 & -0.10 & -0.07 & 0.16 & -0.02 \\
\hline CML322 & -0.10 & -0.05 & -11.16 & -0.02 & 0.05 & 0.05 & 0.19 & -0.09 \\
\hline CML343 & 0.02 & $-0.11^{*}$ & -13.33 & -0.10 & -0.06 & -0.11 & -0.20 & -0.01 \\
\hline CML5 & 0.02 & 0.01 & -14.77 & -0.05 & -0.06 & -0.15 & -0.35 & 0.02 \\
\hline $\mathrm{Hi} 27$ & 0.01 & 0.03 & -16.23 & 0.05 & 0.19 & 0.18 & 0.02 & 0.05 \\
\hline $\mathrm{Ki} 3$ & -0.05 & -0.01 & -14.51 & -0.04 & -0.13 & -0.03 & -0.12 & 0.05 \\
\hline MP705 & -0.06 & -0.01 & -15.58 & 0.07 & 0.03 & 0.02 & -0.01 & 0.08 \\
\hline MP715 & $-0.11^{* *}$ & 0.01 & 45.09 & 0.05 & -0.13 & -0.03 & 0.12 & -0.03 \\
\hline MP719 & -0.01 & 0.05 & -15.86 & 0.03 & $0.26^{*}$ & 0.16 & $0.41^{*}$ & $0.25 * *$ \\
\hline NC298 & -0.01 & 0.01 & -12.57 & -0.01 & -0.02 & -0.02 & -0.05 & 0.02 \\
\hline NC356 & 0.04 & -0.04 & -13.89 & -0.01 & -0.06 & -0.08 & 0.02 & 0.01 \\
\hline TZI8 & $-0.19^{* *}$ & -0.02 & -14.59 & -0.03 & 0.05 & 0.06 & -0.39 & -0.14 \\
\hline $\mathrm{SE} \pm$ & 0.06 & 0.05 & 41.10 & 0.03 & 0.10 & 0.09 & 0.21 & 0.08 \\
\hline \multicolumn{9}{|c|}{ FEMALE GCA } \\
\hline ENTRY-5 & 0.01 & -0.02 & -17.05 & 0.02 & -0.11 & -0.08 & 0.09 & 0.03 \\
\hline ENTRY-6 & - & -0.05 & $74.66^{*}$ & $-0.08 *$ & -0.01 & -0.06 & 0.28 & -0.04 \\
\hline ENTRY-70 & - & 0.04 & -13.79 & 0.02 & 0.01 & -0.02 & 0.18 & -0.03 \\
\hline ENTRY-85 & 0.02 & -0.01 & -17.72 & 0.01 & -0.02 & -0.07 & 0.05 & -0.03 \\
\hline TZEEI-15 & $0.04 *$ & 0.02 & -12.93 & 0.03 & 0.08 & 0.15 & -0.32 & 0.01 \\
\hline TZEEI-6 & 0.01 & 0.03 & -14.62 & 0.01 & 0.05 & 0.08 & -0.30 & 0.06 \\
\hline $\mathrm{SE} \pm$ & 0.02 & 0.03 & 33.33 & 0.05 & 0.05 & 0.08 & 0.23 & 0.03 \\
\hline
\end{tabular}

Note. ${ }^{*}, * *$, Significant at 0.05 and 0.01 probability levels. SE \pm : standard error; -: Missing data.

Further analysis revealed significant genotype by environment interaction for all traits except days to $50 \%$ silking, which suggest that most of the hybrids had similar silking period across sites. Environmental effect on male GCA was significant for all traits as was that of female GCA except for ear rot. Interaction between environments and hybrids was significant for grain yield, anthesis silking interval and plant aspect, but did not significantly influence the rest of the traits (Table 2).

The mean grain yields obtained across the six environments ranged from $4732.12 \mathrm{~kg} / \mathrm{ha}$ (TZEEI- $6 \times$ MP705) to $7588.96 \mathrm{~kg} / \mathrm{ha}($ TZEEI-15 $\times$ MP715) (Table 4). Aflatoxin accumulation in the hybrids ranged from $10.80 \mathrm{ppb}$ $($ ENTRY-5 $\times$ Ki3) to $17.20 \mathrm{ppb}($ ENTRY-5 $\times$ TZI8) among the best fifteen aflatoxin resistant hybrids while the worst hybrid recorded $348.00 \mathrm{ppb}($ TZEEI-15 $\times \mathrm{Ki3}$ ) (Table 4). The various ranges of means for selected traits studied can be found in Table 4 . 
Table 4. Means of 20 of selected aflatoxin accumulation resistant hybrids across six environments

\begin{tabular}{|c|c|c|c|c|c|c|c|c|c|}
\hline Genotype & $\begin{array}{l}\text { Grain } \\
\text { Yield } \\
(\mathrm{Kg} / \mathrm{ha})\end{array}$ & $\begin{array}{l}\text { Ear per } \\
\text { Plant }\end{array}$ & $\begin{array}{l}\text { Cob } \\
\text { Aspect } \\
(1-5) \\
\end{array}$ & $\begin{array}{l}\text { Plant } \\
\text { Aspect } \\
(1-5) \\
\end{array}$ & Stay-Green & $\begin{array}{l}\text { Insect } \\
\text { Damage } \\
(1-5)\end{array}$ & $\begin{array}{l}\text { Ear rot } \\
(1-5)\end{array}$ & $\begin{array}{l}\text { Aflatoxin } \\
\ln (Y+1)\end{array}$ & $\begin{array}{l}\text { Geometric } \\
\text { Means } \\
(\mathrm{ppb})\end{array}$ \\
\hline ENTRY-5 × Ki3 & 7100.49 & 0.92 & 2.01 & 1.86 & 29.50 & 1.31 & 1.53 & 2.38 & 10.80 \\
\hline TZEEI-15 × MP715 & 7575.87 & 0.79 & 2.29 & 2.16 & 44.07 & 1.42 & 1.26 & 2.41 & 11.09 \\
\hline ENTRY-85 × CML247 & 7588.96 & 0.94 & 1.64 & 1.69 & 34.95 & 1.36 & 1.36 & 2.47 & 11.86 \\
\hline ENTRY-85 × Hi27 & 5765.09 & 0.96 & 2.12 & 2.46 & 30.90 & 1.20 & 1.53 & 2.48 & 11.98 \\
\hline TZEEI-6 $\times$ CML247 & 7277.52 & 0.92 & 1.57 & 1.82 & 34.70 & 1.36 & 1.44 & 2.53 & 12.55 \\
\hline TZEEI-15 × CML343 & 7089.82 & 0.99 & 1.40 & 1.67 & 34.23 & 1.12 & 1.42 & 2.52 & 12.37 \\
\hline TZEEI-15 $\times$ Hi27 & 5122.06 & 0.84 & 1.96 & 2.02 & 34.06 & 1.37 & 1.22 & 2.52 & 12.46 \\
\hline ENTRY-85 × CML176 & 6191.14 & 0.94 & 2.02 & 1.77 & 33.30 & 1.20 & 1.50 & 2.53 & 12.52 \\
\hline TZEEI-6 × CML176 & 5698.55 & 0.90 & 1.62 & 2.03 & 37.20 & 1.10 & 1.45 & 2.53 & 12.54 \\
\hline TZEEI- $6 \times$ CML5 & 5519.29 & 1.12 & 2.05 & 2.16 & 37.57 & 1.38 & 1.92 & 2.54 & 12.64 \\
\hline TZEEI-6 × MP705 & 4732.12 & 0.83 & 2.37 & 2.26 & 34.68 & 1.35 & 1.42 & 2.76 & 15.77 \\
\hline TZEEI-6 × CML11 & 7352.49 & 0.99 & 1.62 & 1.60 & 29.16 & 1.39 & 1.34 & 2.79 & 16.34 \\
\hline ENTRY-85 × MP705 & 5913.66 & 1.06 & 2.09 & 2.07 & 33.99 & 1.39 & 1.60 & 2.80 & 16.47 \\
\hline ENTRY-85 × Ki3 & 6105.63 & 1.03 & 1.85 & 1.86 & 31.61 & 1.15 & 1.71 & 2.84 & 17.13 \\
\hline ENTRY-5 $\times$ TZI 8 & 7494.40 & 0.96 & 1.53 & 1.55 & 31.77 & 1.12 & 1.26 & 2.84 & 17.20 \\
\hline AFRIYIE (CHECK) & 6586.30 & 0.91 & 1.78 & 1.82 & 28.41 & 1.10 & 1.35 & 3.35 & 28.60 \\
\hline ENTRY-5 × NC298 & 4248.53 & 0.97 & 2.29 & 1.95 & 32.06 & 1.55 & 1.62 & 5.49 & 241.74 \\
\hline ENTRY-85 × CML11 & 5713.44 & 0.90 & 1.87 & 1.84 & 26.93 & 1.31 & 1.22 & 5.62 & 277.14 \\
\hline TZEEI-15 × MP705 & 4108.30 & 0.91 & 2.14 & 1.71 & 36.72 & 1.51 & 1.74 & 5.78 & 323.30 \\
\hline TZEEI-15 × NC356 & 5204.68 & 0.88 & 2.09 & 2.04 & 33.33 & 1.21 & 1.70 & 5.84 & 342.18 \\
\hline TZEEI-15 $\times$ Ki3 & 5793.83 & 0.96 & 1.71 & 1.75 & 39.61 & 1.18 & 1.29 & 5.85 & 348.00 \\
\hline Mean & 6079.79 & 0.94 & 1.91 & 1.91 & 34.02 & 1.30 & 1.48 & 3.38 & 87.08 \\
\hline Min & 4108.30 & 0.79 & 1.40 & 1.54 & 26.92 & 1.10 & 1.22 & 2.38 & 10.80 \\
\hline Max & 7588.95 & 1.12 & 2.40 & 2.50 & 44.07 & 1.55 & 1.92 & 3.47 & 348.00 \\
\hline $\operatorname{LSD}(0.05)$ & 2038.16 & 0.54 & 0.44 & 0.56 & 122.60 & 0.36 & 1.14 & 203.80 & 203.80 \\
\hline
\end{tabular}

To select hybrids that combined all suitable agronomic traits with reduced aflatoxin accumulation across the six environments, the top twenty hybrids were identified using a base index value (Table 5). Significant $(\mathrm{P}<0.05)$ differences were observed among the genotypic performance for all traits evaluated. Entry- $5 \times$ Tzi8 ranked highest with a score of 5.89 and TZEEI-15 $\times$ MP705 being the least within the top 25 hybrids, with a score of -12.9. It must be noted that some of the hybrid checks outperformed treatments based on the base index rankings (Table 5). 
Table 5. Means of selected traits of 25 hybrids and their base index values across the six environments

\begin{tabular}{|c|c|c|c|c|c|c|c|c|c|c|}
\hline Genotype & $\begin{array}{l}\text { Grain Yield } \\
(\mathrm{Kg} / \mathrm{ha})\end{array}$ & $\begin{array}{l}\text { Ear Per } \\
\text { Plant }\end{array}$ & $\begin{array}{l}\mathrm{CA} \\
(1-5)\end{array}$ & $\begin{array}{l}\mathrm{PA} \\
(1-5)\end{array}$ & Stay-green & $\operatorname{INSD}(1-5)$ & $\begin{array}{l}\text { Aflatoxin } \\
\ln (Y+1)\end{array}$ & $\begin{array}{l}\text { Geometric } \\
\text { means (ppb) }\end{array}$ & $\begin{array}{l}\text { Ear rot } \\
(1-5)\end{array}$ & $\begin{array}{l}\text { BASE } \\
\text { INDEX }\end{array}$ \\
\hline ENTRY-5 × Tzi8 & 7494.40 & 0.96 & 1.53 & 1.55 & 31.77 & 1.12 & 2.84 & 17.20 & 1.26 & 5.89 \\
\hline TZEEI-6 $\times$ CML11 & 7352.49 & 0.99 & 1.62 & 1.60 & 29.16 & 1.39 & 2.79 & 16.34 & 1.34 & 5.89 \\
\hline ENTRY-85 × CML247 & 7588.96 & 0.94 & 1.64 & 1.69 & 34.95 & 1.36 & 2.47 & 11.86 & 1.36 & 5.12 \\
\hline TZEEI-15 × CML343 & 7089.82 & 0.99 & 1.40 & 1.67 & 34.23 & 1.12 & 2.51 & 12.37 & 1.42 & 5.09 \\
\hline ENTRY-85 × Tzi8 & 7002.90 & 1.00 & 1.63 & 1.55 & 30.22 & 1.18 & 3.26 & 26.01 & 1.26 & 4.88 \\
\hline ENTRY-5 × CML- 287 & 7439.64 & 0.99 & 1.62 & 1.61 & 32.70 & 1.20 & 3.08 & 21.74 & 1.34 & 4.73 \\
\hline ENTRY-85 × CML5 & 6647.65 & 1.10 & 1.64 & 1.67 & 35.61 & 1.15 & 3.39 & 29.88 & 1.31 & 3.23 \\
\hline ENTRY-5 × Ki3 & 7100.49 & 0.92 & 2.01 & 1.86 & 29.50 & 1.31 & 2.38 & 10.80 & 1.53 & 2.85 \\
\hline ENTRY-85x CML158 & 5992.12 & 1.02 & 1.62 & 1.84 & 32.57 & 1.01 & 3.05 & 21.27 & 1.27 & 2.70 \\
\hline ENTRY-5 × CML11 & 7511.49 & 1.00 & 1.81 & 1.91 & 33.92 & 1.28 & 3.33 & 28.06 & 1.46 & 2.65 \\
\hline TZEEI-6 × CML247 & 7277.52 & 0.92 & 1.57 & 1.82 & 34.70 & 1.36 & 2.48 & 11.98 & 1.44 & 2.58 \\
\hline TZEEI-6 × CML287 & 6530.83 & 1.03 & 1.95 & 1.50 & 32.06 & 1.38 & 3.05 & 21.06 & 1.37 & 1.64 \\
\hline AFRIYIE (CHECK) & 6586.30 & 0.91 & 1.78 & 1.82 & 28.41 & 1.10 & 3.35 & 28.60 & 1.35 & 1.50 \\
\hline TZEEI-6 × CML343 & 6358.89 & 1.25 & 1.96 & 1.50 & 40.17 & 1.11 & 3.11 & 22.54 & 1.44 & 1.19 \\
\hline TZEEI-6 × Tzi 8 & 6796.41 & 1.13 & 1.96 & 1.47 & 35.42 & 1.26 & 5.19 & 180.51 & 1.40 & 0.77 \\
\hline ENTRY-5 × CML343 & 5695.47 & 0.93 & 1.65 & 1.60 & 32.48 & 1.14 & 2.92 & 18.71 & 1.28 & 0.65 \\
\hline ENTRY-5 × CML5 & 6668.73 & 0.94 & 1.91 & 1.92 & 28.32 & 1.21 & 2.94 & 18.88 & 1.50 & 0.31 \\
\hline TZEEI-15 × MP715 & 7575.87 & 1.03 & 1.76 & 1.76 & 37.91 & 1.22 & 3.94 & 51.67 & 1.48 & 0.00 \\
\hline AHODZIN (CHECK) & 6169.86 & 0.95 & 1.88 & 1.90 & 30.55 & 1.21 & 3.47 & 32.34 & 1.46 & -0.30 \\
\hline ENTRY-85 × NC356 & 6004.39 & 0.93 & 1.80 & 1.79 & 30.18 & 1.35 & 4.69 & 109.65 & 1.43 & -0.44 \\
\hline ENTRY-85 × MP719 & 6247.98 & 0.85 & 2.20 & 1.99 & 34.78 & 1.35 & 4.69 & 101.52 & 1.64 & -7.75 \\
\hline TZEEI-15 × NC356 & 5204.68 & 0.88 & 2.09 & 2.04 & 33.33 & 1.21 & 5.83 & 342.18 & 1.70 & -7.93 \\
\hline ENTRY-5 × NC298 & 4248.53 & 0.97 & 2.29 & 1.95 & 32.06 & 1.55 & 5.48 & 241.74 & 1.62 & -8.32 \\
\hline TZEEI-15 × NC298 & 4438.23 & 0.92 & 1.87 & 1.90 & 41.37 & 1.19 & 5.13 & 170.33 & 1.73 & -8.52 \\
\hline TZEEI-15 $\times$ MP705 & 4108.30 & 0.91 & 2.14 & 1.71 & 36.72 & 1.51 & 5.78 & 323.30 & 1.74 & -12.9 \\
\hline Mean & 6395.93 & 0.98 & 1.81 & 1.74 & 33.32 & 1.25 & 3.64 & 74.78 & 1.45 & \\
\hline Min & 4108.30 & 0.85 & 1.40 & 1.47 & 28.32 & 1.01 & 2.37 & 10.80 & 1.26 & \\
\hline Max & 7588.96 & 1.25 & 2.29 & 2.04 & 41.37 & 1.55 & 5.83 & 342.18 & 1.74 & \\
\hline SED & 1019.08 & 0.27 & 0.22 & 0.28 & 61.30 & 0.18 & 0.57 & 101.90 & 0.20 & \\
\hline
\end{tabular}

Note. SED = standard error of difference; $(\mathrm{ppb})=$ parts per billion.

\subsection{Relative Contributions of Combining Ability Effects}

Heritability estimates of traits largely depend on the relative contributions of all genetic effects compared to non-genetic effects, and narrow-sense heritability specifically depends on additive vs. non-additive genetic effects. The relative proportion and importance of GCA effects was determined as the ratio of GCA effects to total genetic effects using the sum of squares. A close to one means greater predictability based on GCA and a better ability of those plants to form hybrids with many other lines. In contrast, more SCA indicates only specific hybrid combinations will be successful (Baker, 1978). The total contribution of GCA [GCA $m$ plus $\mathrm{GCA}_{\mathrm{f}}$ )] to the total variation among hybrids across the six environments varied from $65 \%$ for husk cover to $96 \%$ for days to $50 \%$ pollen and silking. In general, high GCA for genotypic sum of squares were observed for most of the traits evaluated, particularly for days to $50 \%$ silking and pollen, grain yield, leaf blight and maize streak virus disease resistance. For grain yield, it was evident that GCA sum of squares for male and female were relatively similar although the contribution from the female was slightly higher (Figures 1a and 1b). The general combining ability sum of squares for the female was twice that of the male for traits such as anthesis silking interval and three times more for days to $50 \%$ silking and pollen, stay green, leaf blight and maize streak virus disease resistance (Figures 1a and b). Meanwhile, traits such as cob aspect, plant aspect, leaf rust and ear rot were dominated by higher GCA for genotypic sum of squares for males (Figures 1a and 1b).

On the whole, the genotypic sum of squares for SCA of all traits was lower than GCA, and SCA varied from as low as $4 \%$ for days to $50 \%$ pollen and silking to a maximum of $\sim 73 \%$ for aflatoxin accumulation resistance 
(Figures 1a and 1b). While total GCA for control of aflatoxin accumulation resistance was lower than SCA, the GCA effect of the male was significantly higher than that of the female. This suggests that the heterosis for aflatoxin resistance is not only partitioned but it must be exploited carefully since there was no real variation for resistance in the females, rather than being contributed by one parent to all hybrids formed with it.

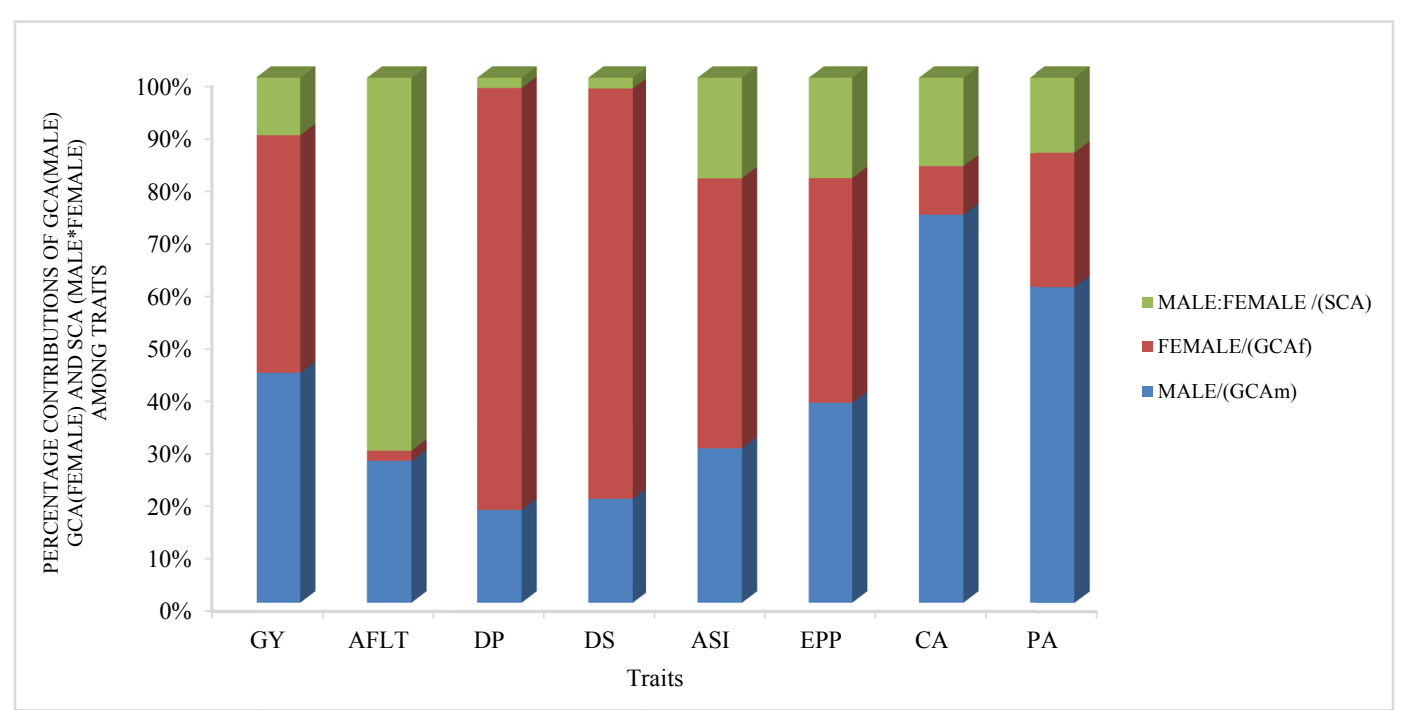

Figure 1a. Proportion of total genotypic sum of squares for grain yield and other agronomic traits of inbred lines attributable to GCA (male), GCA (female) and SCA across 6 environments. GY $=$ Grain yield, AFLT $=$ Aflatoxin accumulation, $\mathrm{DP}=$ days to $50 \%$ Pollen, $\mathrm{DS}=$ Days to $50 \%$ silking, $\mathrm{ASI}=$ anthesis silking interval, $\mathrm{EPP}=$ Ear per plant, $\mathrm{CA}=\mathrm{Cob}$ aspect and $\mathrm{PA}=$ Plant aspect

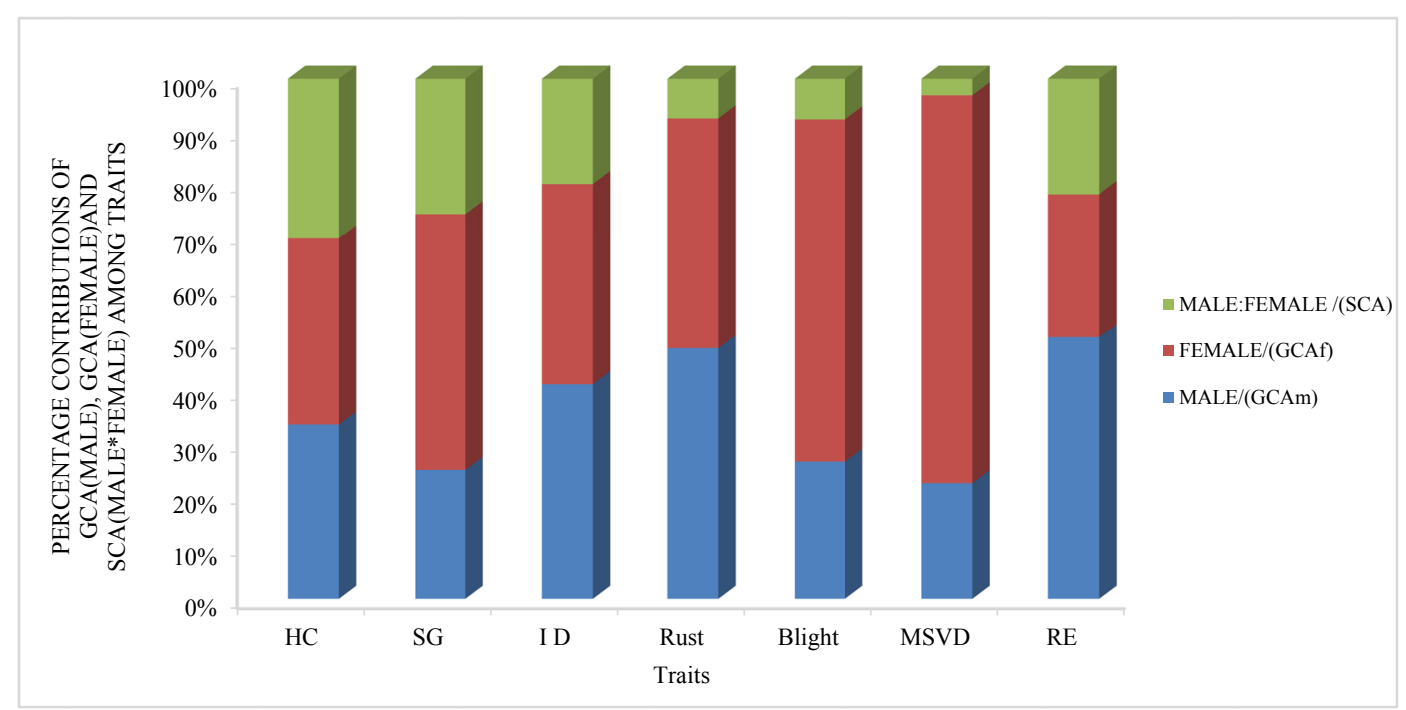

Figure 1b. Proportion of total genotypic sum of squares of grain yield and other agronomic traits of inbred lines attributable to GCA (male), GCA (female) and SCA across 6 environments. $\mathrm{HC}=$ Husk cover $\mathrm{SG}=$ Stay green, $\mathrm{ID}=$ Insect damage, Rust, Blight, MSVD = Maize streak virus disease and Ear rot

\subsection{Heterosis for Grain Yield and Aflatoxin Resistance Across Locations}

Results of mid- and best-parent heterosis estimated for the best performing hybrids are presented in Table 6. The lower or (more negative) values for aflatoxin resistance are the desired outcome for breeding. Among the hybrids, mid-parent heterosis for aflatoxin accumulation resistance varied from $-59.52 \%$ for ENTRY-5 $\times$ Ki3 to $-20.57 \%$ for TZEEI- $6 \times$ TZI8 with a mean of $-36.59 \%$. A total of nine $(45 \%)$ of the best performing hybrids recorded means below the general mean of the mid-parent heterosis. Best-parent heterosis for aflatoxin accumulation 
resistance ranged from a minimum of -57.79 for ENTRY- $5 \times$ Ki3 to a maximum of -18.64 for ENTRY- $85 \times$ CML5 with a mean of -32.40 ; half of the twenty best performing hybrids had heterosis values below this.

Mid-parent heterosis for grain yield of best performing hybrids varied from $325.41 \%$ for ENTRY- $85 \times$ CML158Q to $691.27 \%$ for TZEEI-6 $\times$ CML11 with a mean of 464.08 . Best-parent heterosis also ranged from a minimum of 233.93 to a maximum of $670.37 \%$ for ENTRY- $85 \times$ CML158Q and TZEEI- $6 \times$ CML11, respectively. Forty percent (40\%) of the hybrids produced a higher best-parent heterosis than the mean of 376.32 (Table 6). In general, the high levels of heterosis observed gives an indication of the extent of genetic diversity existing among the genotypes that can be exploited for breeding gain. Additional analysis performed to determine the relationships between aflatoxin accumulation and other agronomic traits revealed a range of weak to moderately strong correlations (Table 7). Aflatoxin accumulation levels were found to correlate negatively and significant $(\mathrm{P}<0.05)$ with grain yield, cob aspect and plant aspect, while it correlated positively but not significant with leaf blight, ear rot and insect damage (Table 7).

Table 6. Mid and High Parent heterosis for aflatoxin accumulation resistance and grain yield of 20 best single cross hybrids evaluated across six environments

\begin{tabular}{|c|c|c|c|c|}
\hline \multirow{3}{*}{$\begin{array}{l}\text { Genotype } \\
\text { Single Crosses }\end{array}$} & \multicolumn{4}{|c|}{ Percentage Heterosis } \\
\hline & \multicolumn{2}{|c|}{ (Aflatoxin resistance control) } & \multicolumn{2}{|c|}{ (Grain Yield) } \\
\hline & Mid-parent & High Parent & Mid-parent & High Parent \\
\hline ENTRY-5 × TZI8 & -38.20 & -37.35 & 542.94 & 407.80 \\
\hline TZEEI-6 × CML11 & -41.50 & -39.86 & 691.27 & 670.37 \\
\hline ENTRY-85 × CML247 & -49.52 & -45.14 & 367.41 & 322.91 \\
\hline TZEEI-15 × CML343 & -50.76 & -41.60 & 345.63 & 308.41 \\
\hline ENTRY-5 × TZI8 & -32.28 & -26.77 & 428.54 & 290.25 \\
\hline ENTRY-85 × CML287 & -23.64 & -20.62 & 495.03 & 404.09 \\
\hline ENTRY-85 × CML5 & -21.91 & -18.64 & 362.18 & 270.46 \\
\hline ENTRY-5 × Ki3 & -59.52 & -57.79 & 456.70 & 381.11 \\
\hline ENTRY-85 × CML158 & -31.41 & -31.23 & 325.41 & 233.93 \\
\hline ENTRY-5 × CML11 & -35.58 & -35.43 & 518.93 & 408.96 \\
\hline TZEEI-6 × CML247 & -41.33 & -40.81 & 516.80 & 400.94 \\
\hline TZEEI-6 × CML 287 & -30.94 & -21.19 & 576.17 & 537.32 \\
\hline TZEEI-15 × CML11 & -40.63 & -33.57 & 390.17 & 279.40 \\
\hline TZEEI-6 × CML343 & -28.98 & -22.74 & 440.50 & 339.76 \\
\hline TZEEI-6 × TZI8 & -20.27 & -19.19 & 671.26 & 649.33 \\
\hline ENTRY-5 × CML343 & -31.78 & -27.91 & 369.29 & 285.91 \\
\hline ENTRY-5 × CML5 & -29.38 & -27.85 & 421.39 & 351.86 \\
\hline ENTRY-5 × CML322 & -33.58 & -30.00 & 399.24 & 329.73 \\
\hline ENTRY-5 × CML176 & -38.67 & -35.96 & 404.91 & 318.05 \\
\hline TZEEI-15 × MP715 & -52.00 & -34.50 & 558.00 & 336.00 \\
\hline Mean & -36.59 & -32.40 & 464.08 & 376.32 \\
\hline Min & -59.52 & -57.79 & 325.41 & 233.93 \\
\hline Max & -20.27 & -18.64 & 691.27 & 670.37 \\
\hline
\end{tabular}

Table 7. Percentage correlation between aflatoxin accumulation and other agronomic traits

\begin{tabular}{llllllllll}
\hline & $\begin{array}{l}\text { Grain } \\
\text { yield }\end{array}$ & Blight & $\begin{array}{l}\text { Cob } \\
\text { Aspect }\end{array}$ & Stay-Green & $\begin{array}{l}\text { Ear per } \\
\text { plant }\end{array}$ & Ear rot & $\begin{array}{l}\text { Insect } \\
\text { damage }\end{array}$ & Plant Aspect & Husk cover \\
\hline Aflatoxin conc. $\ln (\mathrm{Y}+1)$ & -33.00 & 2.00 & -11.00 & -6.00 & -7.00 & 9.00 & 3.25 & -18.00 & -10.00 \\
$\mathrm{P}<(0.05)$ & 0.01 & $\mathrm{~ns}$ & 0.04 & $\mathrm{~ns}$ & $\mathrm{~ns}$ & $\mathrm{~ns}$ & $\mathrm{~ns}$ & 0.02 & $\mathrm{~ns}$ \\
\hline
\end{tabular}

\section{Discussion}

The presence of significant differential environment effects on majority of the traits measured in the current study indicates that the growing environment of germplasms will affect how traits are expressed. Aflatoxin 
contaminations happen when there are environmental situations which affect genotypes especially if these genotypes are susceptible. Significant genotypic difference shows that there was adequate genetic variability among the germplasm to allow for genetic enhancement through selection (Badu-Apraku et al., 2011b; Okoth et al., 2017). The significant GCA and SCA detected for aflatoxin accumulation resistance and most of the other traits represents an opportunity for improvement via selection and offer opportunity for rapid improvement for hybrid variety development.

The significant GCA $\times$ environment interaction observed in this study for aflatoxin accumulation resistance and several other traits has also been reported by Badu-Apraku et al. (2011), Mageto et al. (2017) who highlighted the need to evaluate hybrids and their parental lines in several contrasting environments in order to identify the most promising hybrid combinations for specific traits for each environment. This suggests that more hybrids, each adapted to its own environment, should be offered to farmers, along with recommendations on where each hybrid is expected to perform, rather than a single high yielding hybrid that is expected to do well in all environments, and is unlikely to be found. Farmer variety trials could increase information and adoption of hybrids and could be the next step in the process of creating and disseminating high yielding, aflatoxin accumulation resistant lines for Ghana.

Mid-parent and high-parent heterosis for aflatoxin accumulation resistance among the selected hybrids were -36.39 and -32.40 while that for grain yield were 464.08 and 376.32 respectively. This observation was similar in magnitude to that reported by Saleh et al. (2002) in a study involving tropical single cross, double cross and three-way cross maize hybrids but differed in magnitude from the findings of Betran et al. (2003) who reported average high parent heterosis more than $1225 \%$. In addition, heritability estimates for grain yield was higher $(53.93 \%)$ than for all other traits except aflatoxin accumulation resistance (Table 2). In addition, several traits were negatively correlated to both grain yield and aflatoxin accumulation resistance, which will complicate selection of superior parental lines. Use of a base index for selecting the best performing genotypes may allow gain from selection to be achieved over time for all traits (Menkir et al., 2006; Badu-Apraku et al., 2011a). The use of a base index for identification of parents in this work has been very useful in identifying which of the hybrids combined aflatoxin accumulation resistance and good agronomic characteristics with high yields, thus identifying potential parents of good performing hybrids for consumers to manage the issue of aflatoxin contamination in maize within a limited time.

Nine hybrids were identified across the six environments with consistently good agronomic traits and reduced aflatoxin accumulation based on the base index. They include TZEEI-15 $\times$ CML343, TZEEI- $6 \times$ CML247, ENTRY-85 × Tzi8, ENTRY-5 $\times$ TZI8, TZEEI- 6 CML11, ENTRY-85 $\times$ CML-158Q, ENTRY-5 $\times$ CML-287, TZEEI-6 $\times$ CML343 and TZEEI-15 $\times$ MP715. In addition, while some of the local hybrid checks performed better than some of the crosses, most of the hybrids outperformed the checks (Table 5). The range of aflatoxin accumulation resistance measured in the selected hybrids is comparable to the range recorded by William and Windham (2015) and Brown et al. (2016), but the upper range for the present study is lower than the previous studies. This is quite significant, because the pathogen pressure applied in this study was very high. Natural infection by $A$. flavus would have been lower, and aflatoxin levels correspondingly lower too. Another objective of this study was the GCA effect of individual inbreds on control of aflatoxin resistance and other agronomic traits. Mp715 and Tzi8 consistently produced negative GCA results for aflatoxin accumulation resistance, while Ki3, CML247 and Tzi8 produced positive GCA for grain yield. Generally, most of the exotic inbreds used as males consistently produced negative GCA for aflatoxin accumulation resistance, confirming their initial resistance, and their ability to pass that resistance on to hybrids for controlling aflatoxin accumulation. Mid- and high-parent heterosis levels for aflatoxin accumulation resistance observed in this study indicate hybrids ENTRY-5 $\times$ Ki3 and TZEEI-15x MP715 were simultaneously good for both reduced aflatoxin and high grain yield.

\section{Conclusions}

This study evaluated 96 hybrids under different environments to provide information on the heterosis and mode of gene action conditioning aflatoxin resistance and other important agronomic traits. The study identified some high yielding and aflatoxin resistant hybrids across the environments. Inbred lines with consistent positive GCA for grain yield and negative GCA for aflatoxin accumulation control were identified and can be used for aflatoxin resistance breeding. Hybrids with good performance in specific environments can be recommended for on -farm trials by farmers in those environments. Heritability estimates realised for most traits were moderate to high, suggesting potential gains can be made in trait levels via selection. Most of the hybrids measured out-performed the leading checks. 


\section{Acknowledgements}

This research was funded by USAID through the National Academies of Sciences' (NAS) programme for Partnership for enhanced engagement in Research (PEER). Grant No. Project 4-040, 2015 for "the development of high yielding aflatoxin resistant maize for improved nutrition and health in Ghana".

\section{References}

Agbetiameh, D., Ortega-Beltran, A., Awuah, R. T., Atehnkeng, J., Elzein, A., Cotty, P. J., \& Bandyopadhyay, R. (2020). Field efficacy of two atoxigenic biocontrol products for mitigation of aflatoxin contamination in maize and groundnut in Ghana. Biological Control, 150, 104351. https://doi.org/10.1016/j.biocontrol. 2020.104351

AOAC (Association of Official Analytical Chemists). (1984). Official Methods of Analysis (14th ed.). AOAC, Arlington.

Badu-Apraku, B. (2007). Genetic variances and correlations in an early tropical white maize population after three cycles of recurrent selection for Striga resistance. Maydica, 52, 205-217. https://oi.org/10.1016/ j.fcr.2010.12.011

Badu-Apraku, B., \& Akinwale, R. O. (2011). Cultivar evaluation and trait analysis of tropical early maturing maize under Striga-infested and Striga-free environments. Field Crops Research, 121, 186-194.

Badu-Apraku, B., Oyekunle, M., Akinwale, R. O., \& Aderounmu, M. (2013a). Combining ability and genetic diversity of extra-early white maize inbreds under stress and nonstress environments. Crop Science, 53, 9-26. https://doi.org/10.2135/cropsci2012.06.0381

Baker, R. J. (1978). Issues in diallel analysis. Crop Sci., 18, 533-536. https://doi.org/10.2135/cropsci1978. 0011183X001800040001x

Bankole, S., Schollenberger, M., \& Drochner, W. (2006). Mycotoxins in food systems in Sub Saharan Africa. Rev Mycotoxin Res, 22, 163-169. https://doi.org/10.1007/BF02959270

Beck, D. L., Vasal, S. K., \& Crossa, J. (1990). Heterosis and combining ability of CIMMYT's tropical early and intermediate maturity maize germplasm. Maydica, 35, 279-285.

Betran, F. J., Isakeit, T., \& Odvody, G. (2002). Aflatoxin accumulation of white and yellow maize inbreds in diallel crosses. Crop Science, 42(6), 1894-1901. https://doi.org/10.2135/cropsci2002.1894

Betrán, F. J., Ribaut, J. M., Beck, D., \& De León, G. D. (2003). Genetic diversity, specific combining ability, and heterosis in tropical maize under stress and nonstress environments. Crop Science, 43, 797-806. https://doi.org/10.2135/cropsci2003.7970

Brown, R. L., Williams, W. P., Windham, G. L., Menkir, A., \& Chen, Z. Y. (2016). Evaluation of African-Bred Maize Germplasm Lines for Resistance to Aflatoxin Accumulation. Agronomy, 6, 24. https://doi.org/ 10.3390/agronomy6020024

Cairns, J. E., Sonder, K., Zaidi, P. H., Verhulst, N., Mahuku, G., Babu, R., ... Prasanna, B. M. (2012). Maize production in a changing climate: Impacts, adaptation, and mitigation strategies. Adv. Agron., 114, 1-58. https://doi.org/10.1016/B978-0-12-394275-3.00006-7

Chen, Z. Y., Brown, R. L., Cleveland, T. E., Damann, K. E., \& Russin, J. S. (2001). Comparison of constitutive and inducible maize kernel proteins of genotypes resistant or susceptible to aflatoxin production. J. Food Prot., 64, 1785-1792. https://doi.org/10.4315/0362-028X-64.11.1785

Cochran, W. G., \& Cox, G. M. (1960). Experimental designs. John Wiley and Sons, New York, USA.

Cotty, P. J. (1989). Virulence and cultural characteristics of two Aspergillus flavus strains pathogenic on cotton. Phytopathology, 79, 808-814. https://doi.org/10.1094/Phyto-79-808

Daves, A. C., Windham, G. L., \& Williams, P. W. (2010). Aflatoxin Accumulation in Commercial Corn Hybrids Artificially Inoculated with Aspergillus flavus in 2008 and 2009. Research Report, 24(9).

East, E. M. (1936). Heterosis. Genetics, 21, 375-397. https://doi.org/10.1093/genetics/21.4.375

Ewool, M. B. (2004). Estimation of genetic improvement of maize in Ghana under three levels of nitrogen application (Msc Thesis, Kwame Nkrumah University of Science and Technology, Kumasi, Ghana).

FAOSTAT. (2019). FAO statistical database online. Retrieved July 19, 2021, from http://www.fao.org/faostat/ en\#data/QC 
Githang'a, D., Wangia, R. N., Mureithi, M. W., Wandiga, S. O., Mutegi, C., Ogutu, B., ... Anzala, O. (2019). The effects of aflatoxin exposure on Hepatitis B-vaccine induced immunity in Kenyan children. Current Problems in Pediatric and Adolescent Health Care, 49(5), 117-130. https://doi.org/10.1016/j.cppeds. 2019.04.005

Gong, Y. Y., Turner, P. C., Hall, A. J., \& Wild, C. P. (2008). Aflatoxin exposure and impaired child growth in West Africa: An unexplored international public health burden? In J. F. Leslie, R. Bandyopadhyay, \& A. Visconti (Eds.), Mycotoxins. Detection methods, management, public health and agricultural trade (pp. 53-65). CABI, Oxfordshire. https://doi.org/10.1079/9781845930820.0053

Guo, B. Z., Li, R. G., Widstrom, N. W., Lynch, R. E., \& Cleveland, T. E. (2001). Genetic variation within maize population GT-MAS:gk and the relationship with resistance to Aspergiilus flavus and aflatoxin production. Theoretical and Applied Genetics, 103(4), 533-539. https://doi.org/10.1007/s001220100655

Hallauer, A. R., \& Miranda, J. B. (1988). Quantitative genetics in maize breeding (2nd ed.). Iowa State University Press Ames, USA. Retrieved August 6, 2021, from https://knoema.com/atlas/Ghana/topics/ Agriculture/Crops-Production-Yield/Maize-yield

Ifie, B. E. (2013). Genetic analysis of Striga resistance and low soil nitrogen tolerance in early maturing maize (Zea mays L.) inbred lines (PhD Thesis, West Africa Centre for Crop Improvement, School of Agriculture, College of Agriculture and Consumer Sciences, University of Ghana Legon, Ghana).

IITA (International Institute of Tropical Agriculture). (2000). Impact of IITA-improved germplasm on maize production in West and Central Africa. IITA, Ibadan, Nigeria.

IITA (International Institute of Tropical Agriculture). (2009). Maize. IITA, Ibadan, Nigeria. Retrieved September 8, 2014, form http://www.iita.org/maize

IITA (International Institute of Tropical Agriculture). (2013). Annual report 2013. IITA, Ibadan, Nigeria. http://newint.iita.org/wp-content/uploads/2016/04/Annual-Report-2013.pdf

Jha, D. K. (1995). Laboratory Manual on Seed Pathology (pp. 13-30). Vikas Publishing House (PVT) Ltd.

Jolly, P., Jiang, Y., Ellis, W., Awuah, R., Nnedu, O., Phillips, T., ... Jolly, C. (2006). Determinants of aflatoxin levels in Ghanaians: Socio demographic factors, knowledge of aflatoxin and food handling and consumption practices. Int. J. Hyg. Environ. Health., 209(4), 345-58. https://doi.org/10.1016/j.ijheh. 2006.02.002

Kim, S. K. (1994). Genetics of Maize Tolerance of Striga hermonthica. Crop Science, 34, 900-907. https://doi.org/10.2135/cropsci1994.0011183X003400040012x

Klich, M. A., \& Pitt, J. I. (1988). A laboratory guide to common Aspergillus species and their teleo-morphs ( $\mathrm{p}$. 116). CSIRO, North Ryde, New South Wales, Australia.

Kpotor, P. (2012). Evaluation of newly released maize varieties in Ghana for yield and stability under three nitrogen application rates in two agro-ecological zones (Doctoral dissertation, Department of Crop and Soil Sciences, Faculty of Agriculture, College of Agriculture and Natural Resources, Kwame Nkrumah University of Science and Technology, Kumasi, Ghana).

Liang, X. Q., Luo, M., \& Guo, B. O. (2006). Resistance mechanisms to Aspergillus flavus infection and aflatoxin contamination in peanuts (Arachis hypogaea). Plant Pathol J, 5, 115-124. https://doi.org/10.3923/ ppj.2006.115.124

Mageto, E. K., Makumbi, D., Njoroge, K., \& Nyankanga, R. (2017). Genetic analysis of early-maturing maize (Zea mays L.) inbred lines under stress and nonstress conditions. J. of Crop Improvement, 31(4), 560-588. https://doi.org/10.1080/15427528.2017.1315625

Matacic, C. (2016). Fungal toxins are poisoning Africa's children, says new report. Science, AAAS, USA. https://doi.org/10.1126/science.aaf4085

Mayfield, K. L., Murray, S. C., Rooney, W. L., Isakeit, T., \& Odvody, G. A. (2011). Confirmation of QTL reducing aflatoxin in maize test crosses. Crop Science, 51(6), 2489-2498. https://doi.org/10.2135/ cropsci2011.02.0112

Mayfield, K., Betran, F. J., \& Isakeit, T. (2012). Registration of maize germplasm lines Tx736, Tx739, and Tx740 for reducing preharvest aflatoxin accumulation. Journal of Plant Registrations, 6(1), 88-94. https://doi.org/10.3198/jpr2010.12.0675crg 
McMillian, W. W., Widstrom, N. W., \& Wilson, D. M. (1993). Registration of GT-MAS:gk.maize germplasm. Crop Science, 33, 882. https://doi.org/10.2135/cropsci1993.0011183X003300040064x

Menkir, A., Olowolafe, M. O. Ingelbrecht, I., Fawole, I., Badu-Apraku, B., \& Vroh, I. (2006). Assessment of testcross performance and genetic diversity of yellow endosperm maize lines derived from adapted and exotic backcrosses. Theor. Appl. Genet., 113, 90-99. https://doi.org/10.1007/s00122-006-0275-5

Miller, J. O. (1996). Mycotoxins. In K. F. Cardwell (Ed.), Proceedings of the workshop on mycotoxins in food in Africa (pp. 18-22). Cotonou, Benin.

Odindo, B. A. (2017). Genetic analysis of resistance to aspergillus ear rot and aflatoxin accumulation in maize inbred lines (A Master's thesis research submitted to the department of plant science and crop protection, Faculty of agriculture, university of Nairobi, Kenya).

Okoth, S., Rose, L. J., Ouko, A., Beukes, I., Sila, H., Mouton, M., ... Viljoen, A. (2017). Field evaluation of resistance to aflatoxin accumulation in maize inbred lines in Kenya and South Africa. Journal of Crop Improvement, 31(6), 862-878. https://doi.org/10.1080/15427528.2017.1391915

Perrone, G., Miriam, H., Gaetano S., Filomena E., Ranajit, B., John, F. L., \& Logrieco, A. (2014). Population structure and Aflatoxin production by Aspergillus Sect. Flavi from maize in Nigeria and Ghana. Food Microbiology, 41, 52-59. https://doi.org/10.1016/j.fm.2013.12.005

Powers, L. (1944). An expansion of Jones' theory for the explanation of heterosis. Am. Nat., 78, $275-280$. https://doi.org/10.1086/281199

Pswarayi, A., \& Vivek, B. S. (2008). Combining ability amongst CIMMYT's early maturing maize (Zea mays L.) germplasm under stress and non-stress conditions and identification of testers. Euphytica, 162, 353-362. https://doi.org/10.1007/s10681-007-9525-0

Ramjee, G. (1996). Aflatoxin, kwashiorkor and morbidity. In K. F. Cardwell (Ed.), Proceedings of the workshop on mycotoxins in food in Africa (p. 25). Cotonou, Benin.

SAS Institute, Inc. (2012). Statistical analysis software (SAS) user's guide. SAS Institute, Inc., Cary, NC.

Scott, G. E., \& Zummo, N. (1994). Kernel infection and aflatoxin production in maize by Aspergillus flavus relative to inoculation and harvest dates. Plant Dis., 78(2), 123-125. https://doi.org/10.1094/PD-78-0123

Shaw, R. H. (1988). Climate requirements. In G. F. Sprague \& J. W. Dudley (Eds.), Corn and corn improvement (pp. 609-638). Am. Soc. of Agronomy, Madison, Wisconsin, USA. https://doi.org/10.2134/agronmonogr 18.3ed.c10

Shephard, G. S. (2008). Risk assessment of aflatoxins in food in Africa. Food Addit. Contam.: Part A, 25, 1246-1256. https://doi.org/10.1080/02652030802036222

Shull, G. H. (1908). The composition of a field of maize. Journal of Heredity, 4(1), 296-301. https://doi.org/ 10.1093/jhered/os-4.1.296

Wacoo, A. P., Wendiro, D., Vuzi, P. C., \& Hawumba, F. F. (2014). Methods for Detection of Aflatoxins in Agricultural Food Crops. Journal of Applied Chemistry, 2014, Article ID 706291. https://oi.org/10.1155/ 2014/706291

Warburton, M. L., \& Williams, W. P. (2014). Aflatoxin Resistance in Maize: What Have We Learned Lately? Advances in Botany, 2014, Article ID 352831. https://doi.org/10.1155/2014/352831

Warburton, M. L., Williams, W. P., \& Windham, G. (2013). Phenotypic and genetic characterization of a maize association mapping panel developed for the identification of new sources of resistance to Aspergillus flavus and aflatoxin accumulation. Crop Science, 53, 2374-2383. https://doi.org/10.2135/cropsci2012. 10.0616

Widstrom, N. W., Wilson, D. M., \& McMillian. W. W. (1984). Ear resistance of maize inbreds to field aflatoxin contamination. Crop Sci., 24, 1155-1157. https://doi.org/10.2135/cropsci1984.0011183X002400060035x

Wild, C. P., Jiang, Y. D., Allen, S. J., Jansen, L. A. M., Hall, A. J., \& Montesano, R. (1990). Aflatoxin albumin adducts in human sera in different regions of the world. Carcinogenesis, 12, 2271-2274. https://doi.org/ $10.1093 /$ carcin/11.12.2271

Williams, W. (1959). Heterosis and the genetics of complex characters. Nature, 184, 527-530. https://doi.org/ $10.1038 / 184527 \mathrm{a} 0$ 
Williams, W. P., \& Windham, G. L. (2015). Aflatoxin accumulation in a maize diallel cross. Agriculture, 5, 344-52. https://doi.org/10.3390/agriculture5020344

Williams, W. P., Windham, G. L., \& Buckley, P. M. (2008). Diallel analysis of aflatoxin accumulation in maize. Crop Science, 48(1), 134-138. https://doi.org/10.2135/cropsci2007.05.0306

Windham, G. L., Williams, W. P., Buckley, P. M., \& Abbas, H. K. (2003). Inoculation techniques used to quantify aflatoxin resistance in corn. J. Toxicol.-Toxin Reviews, 22, 313-325. https://doi.org/10.1081/ TXR-120024096

Womack, E. D., Williams, W. P., Windham, G. L., \& Xu, W. (2020). Mapping quantitative trait loci associated with resistance to aflatoxin accumulation in maize inbred MP719. Frontiers in Microbiology, 11, 45. https://doi.org/10.3389/fmicb.2020.00045

\section{Copyrights}

Copyright for this article is retained by the author(s), with first publication rights granted to the journal.

This is an open-access article distributed under the terms and conditions of the Creative Commons Attribution license (http://creativecommons.org/licenses/by/4.0/). 